\title{
DEVELOPMENT OF FAULT ACCOMMODATION SYSTEM FOR ACTUATORS OF UNDERWATER MANIPUlators
}

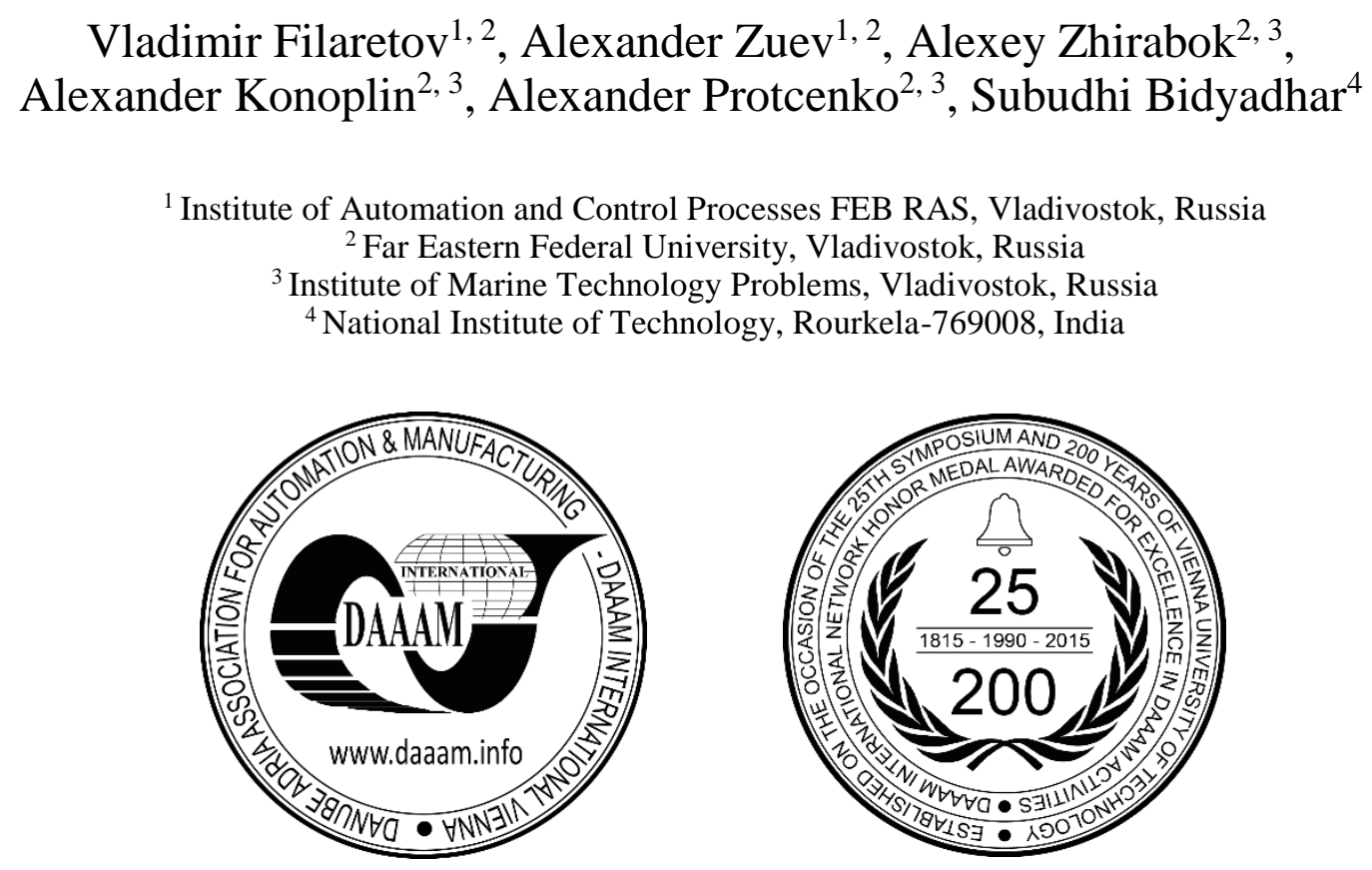

This Publication has to be referred as: Filaretov, V[ladimir]; Zuev, A[lexander]; Zhirabok, A[lexey]; Konoplin, A[lexander]; Protcenko, A[leksandr] \& Subudhi, B[idyadhar] (2017). Development of Fault Accommodation System for Actuators of Underwater Manipulators, Proceedings of the 28th DAAAM International Symposium, pp.0014-0022, B. Katalinic (Ed.), Published by DAAAM International, ISBN 978-3-902734-11-2, ISSN 1726-9679, Vienna, Austria DOI: $10.2507 / 28$ th.daaam.proceedings.002

\begin{abstract}
The paper presents a fault accommodation system for electric servo actuators of underwater manipulators. These actuators are described by nonlinear equations with variable parameters. It is assumed, that the following typical faults are possible in electric servo actuators: fault caused by changing of viscous friction moment and error in the sensor, which measures the position of output shaft of gear. The proposed system is based on using of diagnostic observers with residual signal feedback. It is proposed the methodology of synthesis of such observers for detection and identification of occurred faults. The accommodation to faults is ensured due to the generation of additional control signals based on identified value of faults. This provides the reliability of underwater working of manipulators at faults. The efficiency of the proposed system is confirmed by mathematical modelling.
\end{abstract}

Keywords: underwater manipulator; diagnosis; fault; accommodation; actuators

\section{Introduction}

Now autonomous underwater vehicles (AUV) are the most effective research instrument of the World Ocean and performance of various underwater and technical operations [1,2]. At this, fitting multilink underwater manipulators (UM) on AUVs is a very perspective tendency of development [3-6]. The manipulator-equipped AUVs can be effectively used for fulfillment of research operations in hydrogeology, hydrobiology, geophysics, hydrochemistry, in oil and gaze extraction. In these cases the following operations are carried out: selective sampling of soil probes and geological rocks; collecting bio-organisms; set-up and servicing of different tools; definition of composition and density of the soil using special probes and drills; taking precipitation samples by means of hermetically sealed soil tubes; 
measurements with thermistor sensors in different layers of sedimentary soil etc. All it considerably expands the field of effective use of AUVs and allows to exclude the human at a research of sea depths.

An important task at autonomous operation of the UMs is to increase in their reliability and safety. Any fault in the actuators of UMs leading significant reduction of accuracy and therefore quality of performance. Moreover, at some faulty cases the expensive vehicle can be lost. The task of increase of reliability can be solved by using modern fault diagnosis and accommodation systems [7, 8]. Fault accommodation is based on forming additional control signals for automatic stabilization of main parameters of the actuators when faults occur. For forming of such control signals all possible faults should be timely detected and their values should be estimated.

As a rule, the actuators of UMs are described by essentially nonlinear equations. Therefore, it is necessary to use nonlinear methods of fault diagnosis and accommodation. However, most of papers dealing with this problem consider nonlinear systems with differential nonlinearities $[9,10]$ therefore they cannot be used in our case. Today, a several approaches for fault diagnosis and accommodation for systems with no differentiable nonlinearities are known. Among them there are methods using the algebra of functions [11], the logic-dynamic approach (LDA) [12], and others [13, 14]. Whereas the algebra of functions demands complex analytical calculations using symbolic mathematical packages, the LDA allows using the methods of linear algebra for nonlinear systems and provides relatively simple procedure for synthesis of diagnostic observers. There are several fault accommodation systems based on LDA observers [15, 16]. These systems provide exact faults identification and accommodation for only actuators of manipulators with fully measured state vector. This condition requires installation of additional sensors for actuators of UMs, which in turn reduces reliability and is not always possible constructively.

When UMs move in water a significant generalized moments act in all its degrees of freedom. These moments are caused by inertial and gravitational forces and forces determined by interaction of working UMs and viscous environment [17]. At this case, the actuators of UMs will be described by nonlinear equations with variable parameters. It should be considering at synthesis of diagnostic observers and accommodation control law.

Thus, the problem of development of highly efficient and easily implementing fault accommodation systems for actuators of UMs is still topical.

This paper considers a solution to the problem of development of the effective accommodation system for faults caused by errors in the sensor, which measure the position of output shaft of gear and by changing the value of viscous friction coefficient in electric servo actuators of the UMs described by mathematical model including differential equations with nonlinearity and variable parameters and not fully known state vector.

\section{Description of model of the actuators of UMs}

Let's consider the AUV with UM fixed in point O (see Fig. 1). In general, this UM can have $n$ degrees of freedom. The UM is able to move in forward hemisphere in front of the AUV. Each degree of freedom is actuated by corresponding actuator.

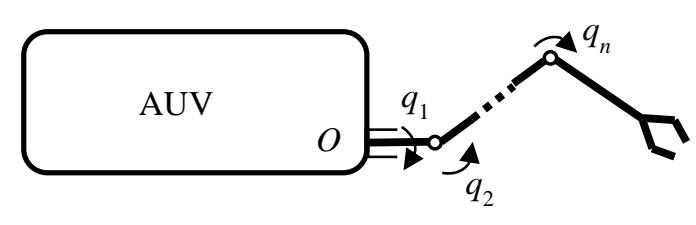

Fig. 1. The AUV with n degrees of freedom UM

For definition of external generalized moment arising in all degrees of freedom of UM at the motion in water, it is possible to use recurrent algorithm for solving inverse dynamics problem of the UM [17]. This algorithm is based on well-known two-staged computational structure. At first stage, according to this structure the calculation of angular speeds and accelerations of centers of weights of the UM links is carried out starting with first and finishing with last the UM link. At second stage, the definition of forces and moments acting on all UM joints is carried out in the reverse order. This algorithm can be written down as [17]

$$
\begin{aligned}
& \omega_{i}=A_{i}^{i-1} \omega_{i-1}+e_{i} \dot{q}_{i} \bar{\sigma}_{i}, \omega_{0}=\omega_{0}^{*}, i=\overline{1, n} ; \\
& \dot{\omega}_{i}=A_{i}^{i-1} \dot{\omega}_{i-1}+\left[\left(A_{i}^{i-1} \omega_{i-1}\right) \times e_{i} \dot{q}_{i}+e_{i} \ddot{q}_{i}\right] \bar{\sigma}_{i}, \dot{\omega}_{0}=\dot{\omega}_{0}^{*}, i=\overline{1, n} ; \\
& \ddot{P}_{i}=A_{i}^{i-1}\left(\ddot{P}_{i-1}+\delta_{i-1} p_{i-1}^{*}\right)+\left(2 \dot{q}_{i} \omega_{i} \times e_{i}+\ddot{q}_{i} e_{i}\right) \sigma_{i}, \ddot{P}_{0}=P_{0}^{*} ; \\
& \ddot{r}_{m i}=\ddot{P}_{i}+\delta_{i} r_{i}^{*}, i=\overline{1, n} ; \\
& \mathrm{v}_{i}=A_{i}^{i-1}\left(\mathrm{v}_{i-1}+\omega_{i-1} \times p_{i-1}^{*}\right), \mathrm{v}_{1}=\mathrm{v}_{0}, i=\overline{2, n} ; \quad \mathrm{v}_{A i}=\mathrm{v}_{i}+\omega_{i} \times r_{i}^{*} ; \\
& \psi_{i}=\arccos \frac{\mathrm{v}_{A i} p_{i}^{*}}{\left|\mathrm{v}_{A i}\right|\left|p_{i}^{*}\right|}, \alpha_{i}^{*}=\arccos \frac{\mathrm{v}_{i} p_{i}^{*}}{\left|\mathrm{v}_{i}\right|\left|p_{i}^{*}\right|}, \beta_{i}^{*}=\arccos \frac{\omega_{i} p_{i}^{*}}{\left|\omega_{i}\right|\left|p_{i}^{*}\right|}, i=\overline{1, n} ;
\end{aligned}
$$




$$
\begin{aligned}
& r_{p i}=r_{i}^{*}+K_{A i} \mathrm{v}_{A i}, i=\overline{1, n} ; \\
& \mathrm{v}_{j}^{*}=\mathrm{v}_{i}+\omega_{i} \times h_{j}^{*}, \omega_{L i}=\left|\omega_{i}\right| \mathrm{e}_{L i} \cos \beta_{i}^{*}, i=\overline{1, n}, j=\overline{1, N} ; \\
& \mathrm{v}_{L i}^{*}=\left|\mathrm{v}_{i}\right| \mathrm{e}_{L i} \cos \alpha_{i}^{*}, \mathrm{v}_{p j}^{*}=\mathrm{v}_{j}^{*}-\mathrm{v}_{L i}^{*}, i=\overline{2, n}, j=\overline{1, N} ; \\
& \text { if } \frac{\rho \mathrm{v}_{L i}^{*} l_{i}}{\eta} \leq 10^{3} \text { then } F_{R L i}=k_{L i} \eta \mathrm{v}_{L i}^{*}, i=\overline{n, 1} ; \\
& \text { if } \frac{\rho \mathrm{v}_{L i}^{*} l_{i}}{\eta}>10^{3} \text { then } F_{R L i}=\frac{1}{2} \rho k_{L i} s_{i} \mathrm{v}_{L i}^{* 2}, i=\overline{n, 1} ; \\
& \text { if } \frac{2 \rho \mathrm{v}_{p i}^{*} r_{i}}{\eta} \leq 10^{3} \text { then } F_{R p j}=k_{i}^{*} \eta_{\mathrm{v}_{p j}^{*}}^{*}, i=\overline{n, 1}, j=\overline{1, N} ; \\
& \text { if } \frac{2 \rho \mathrm{v}_{p i}^{*} r_{i}}{\eta}>10^{3} \text { then } F_{R p j}=\frac{1}{2} \rho k_{i}^{*} d_{j} \mathrm{v}_{p j}^{* 2}, i=\overline{n, 1}, j=\overline{1, N} ; \\
& F_{R p i}=\sum_{j=1}^{N} F_{R p j}, M_{R p j}=h_{j}^{*} \times F_{R p j}, M_{R p i}=\sum_{j=1}^{N} M_{R p j}, M_{L i}=k_{L i}^{*} \eta r_{i} \omega_{L i}, i=\overline{n, 1}, j=\overline{1, N} \\
& F_{i}=A_{i}^{i+1} F_{i+1}+\left(m_{i}+\Pi_{m i}\right) \ddot{r}_{m i}+F_{R L i}+F_{R p i}, F_{n+1}=0, i=\overline{n, 1} ; \\
& M_{i}=A_{i}^{i+1} M_{i+1}+p_{i}^{*} \times\left(A_{i}^{i+1} F_{i+1}\right)+r_{i}^{*} \times\left(m_{i} \ddot{r}_{m i}\right)+r_{p i} \times\left(\Pi \Pi_{m i} \ddot{r}_{m i}\right)+ \\
& +\left(\tau_{i}+T_{i}\right) \dot{\omega}_{i}+\omega_{i} \times\left(\left(\tau_{i}+T_{i}\right) \omega_{i}\right)+M_{R p i}+M_{L i}, M_{n+1}=0, i=\overline{n, 1} ; \\
& \hat{M}{ }_{i}=e_{i}^{\mathrm{T}}\left(F_{i} \sigma_{i}+M_{i} \bar{\sigma}_{i}\right),
\end{aligned}
$$

where $\delta_{i}=\left[\begin{array}{ccc}-\left(\omega_{i(2)}^{2}+\omega_{i(3)}^{2}\right) & \omega_{i(1)} \cdot \omega_{i(2)}-\dot{\omega}_{i(3)} & \omega_{i(1)} \cdot \omega_{i(3)}+\dot{\omega}_{i(2)} \\ \omega_{i(1)} \cdot \omega_{i(2)}+\dot{\omega}_{i(3)} & -\left(\omega_{i(1)}^{2}+\omega_{i(3)}^{2}\right) & \omega_{i(2)} \cdot \omega_{i(3)}-\dot{\omega}_{i(1)} \\ \omega_{i(1)} \cdot \omega_{i(3)}-\dot{\omega}_{i(2)} & \omega_{i(2)} \cdot \omega_{i(3)}+\dot{\omega}_{i(1)} & -\left(\omega_{i(1)}^{2}+\omega_{i(2)}^{2}\right)\end{array}\right]$ (in matrix $\delta_{i}$ lower index indicates number of element in respective vectors); $A_{i}^{i-1}$ is matrix of vectors translation from (i-1)- th to $i$ - th coordinate system; $\mathrm{v}_{0}$ is linear velocity of the UM base; $e_{i}=\left(\begin{array}{lll}0 & 0 & 1\end{array}\right)^{T}$ is vector directed along axis of joint $i ; \omega_{0}^{*} \in R^{3}$ is angular velocity of AUV rotation; $q_{i}$ is angle of joint i rotation; $\omega_{i} \in R^{3}$ is angular velocity of link $i ; p_{i}^{*} \in R^{3}$ is concerned with longitudinal axis of link $\mathrm{i}$ vector defining position of joint $(i+1)$ relative to joint $i ; e_{L i} \in R^{3}$ is unitary vector directed along longitudinal axis of link $i ; h_{j}^{*} \in R^{3}$ is vector defining centre of mass of segment $\mathrm{j}$ of length $\delta h_{i}^{*}$ of link $i$ concerning joint $i ; \alpha_{i}^{*}=\arccos \frac{\mathrm{v}_{i} \cdot p_{i}^{*}}{\left|\mathrm{v}_{i}\right|\left|p_{i}^{*}\right|}$ is an angle between $\mathrm{v}_{i}$ and $p_{i}^{*} ; l_{i}$ and $r_{i}$ are length and radius of link $i$, respectively; $\rho$ and $\eta$ are density and viscosity of water, respectively; $\mathrm{v}$ and $d$ are linear velocity and characteristic size of body, respectively); $\mathrm{v}_{A i} \in R^{3}$ is linear velocity of center of size of UM link $i ; \sigma_{i}=1$ if joint $i$ is translational; $\sigma_{i}=0$ if joint $i$ is rotational $\left(\bar{\sigma}_{i}=1-\sigma_{i}\right) ; m_{i}$ is mass of link $i ; \dot{\omega}_{i} \in R^{3}$ is angular acceleration of link $i ; \omega_{L i} \in R^{3}$ is component of angular velocity $\omega_{i}$ parallel to longitudinal axis of link $i ; \Pi_{m i}$ are added masses of fluid attached to link $i ; \ddot{r}_{m i} \in R^{3}$ is linear acceleration of center of mass of link $i$; $r_{p i} \in R^{3}$ is vector defining position of center of added mass of fluid $\Pi_{m i}$ relative to joint $i ; r_{i}^{*} \in R^{3}$ is vector defining position of center of mass of link relative to joint $i ; k_{L i}^{*}$ is experimentally determined viscous friction coefficient; $K_{A i}$ is parameter depending from $\left|\mathrm{v}_{A i}\right|$ and angle $\psi_{i} ; \tau_{i} \in R^{3 \times 3}$ is inertia tensor of link $i$ concerning its centre of mass; $T_{i} \in R^{3 \times 3}$ is inertia tensor of fluid masses attached to UM link $i ; F_{i}, M_{i} \in R^{3}$ are force and torque acting on joint $i$, respectively; $P_{0}^{*}=-g+\ddot{P}_{U V}$ if centres of mass of UM links do not coincide with their centres of size; $P_{0}^{*}=g\left(\frac{w_{i}}{m_{i}}-1\right)+\ddot{P}_{U V}$ if centres of mass of all UM links coincide with their centres of size; $\ddot{P}_{U V} \in R^{3}$ is linear acceleration of UM base; $g$ is intensity of gravity; $w_{i}$ is mass of fluid forced out by link; $\hat{M}_{i}$ is generalized moment that arises in $i$-th degree of freedom of the UM at the motion in water. Further for simplicity we will not write the index $i$.

Each degree of the UM has an electric servo actuator that includes DC motor, reducing gear and sensors measuring the angular position and angular speed of output shaft of reducing gear.

It is assumed that the following typical faults are possible in actuators of the UM: 
- the fault $d_{1}(t)$ caused by error $\tilde{\alpha}_{r}$ of sensor of angular position;

- the fault $d_{2}(t)$ caused by change of value of viscous friction coefficient $\tilde{k}_{v}$;

Considering these faults and (1), model of each actuator of the UM can be presented by following differential equations:

$$
\begin{aligned}
& \dot{x}_{1}=\frac{1}{i_{r}} x_{2}+d_{1}(t), \\
& \dot{x}_{2}=-\frac{k_{v}}{J} x_{2}+\frac{k_{m}}{J} x_{3}-\frac{M_{c f}+\hat{M}^{*}}{J}+d_{2}(t), \\
& \dot{x}_{3}=-\frac{k_{\omega}}{L} x_{2}-\frac{R}{L} x_{3}+\frac{k_{u}}{L} u,
\end{aligned}
$$

where $x_{1}=\alpha_{r}$ is the angle of output shaft of reducing gear; $x_{2}=\dot{\alpha}$ is the speed of DC motor shaft; $R, L$ and $x_{3}=I$, are the resistance, inductance and current of electric motor rotor circuits, accordingly; $k_{\omega}$ is the coefficient of counter-EMF; $k_{m}$ is the coefficient of motor moment; $k_{v}$ is the coefficient of viscous friction; $J$ is the rotational inertia of rotating parts of reduction drive and DC motor; $i_{r}$ is the coefficient of the reducing gear; $u$ is the control signal; $k_{u}$ is the amplifier gain; $\hat{M}^{*}=\frac{\hat{M}}{i_{r}} ; M_{c f}=M_{c f 0} \operatorname{sign}(\dot{a})$ is moment of Coulomb friction; $M_{c f 0}$ is coefficient of Coulomb friction.

The variables $d_{1}$ and $d_{2}$ in (1) represent the effects of faults on the system and can be presented in next form $d_{1}(t)=\dot{\tilde{\alpha}}_{r}$ $d_{2}(t)=-\frac{\tilde{k_{v}} \dot{\alpha}}{J}$.

It should be noted, that (1) has nonlinearities and significantly changing parameters because of changes in parameter $\hat{M}^{*}$.

Equation (2) can be represented by the differential equation in a matrix form as follows:

$$
\begin{aligned}
& \dot{x}(t)=F x(t)+G u(t)+B(x)+E d, \\
& y(t)=N x(t),
\end{aligned}
$$

where $B(x) \in R^{3}$ is a vector corresponding to the system nonlinearities, $x \in R^{3}, y \in R^{2}, d \in R^{2}, F \in R^{3 \times 3}, G \in R^{3}$, $E \in R^{3 \times 2}, \quad N \in R^{2 \times 3}$

$F=\left[\begin{array}{ccc}0 & 1 / i_{r} & 0 \\ 0 & -k_{v} / J & k_{m} / J \\ 0 & -k_{\omega} / L & -R / L\end{array}\right], \quad x=\left[\begin{array}{c}x_{1} \\ x_{2} \\ x_{3}\end{array}\right], \quad E=\left[\begin{array}{ll}1 & 0 \\ 0 & 1 \\ 0 & 0\end{array}\right], \quad N=\left[\begin{array}{lll}1 & 0 & 0 \\ 0 & 1 & 0\end{array}\right], \quad B(x)=\left[\begin{array}{c}0 \\ -\left(M_{c f}+\hat{M}^{*}\right) / J \\ 0\end{array}\right], \quad G=\left[\begin{array}{c}0 \\ 0 \\ k_{u} / L\end{array}\right]$, $y=\left[\begin{array}{c}\alpha_{r} \\ \dot{\alpha}\end{array}\right], d=\left[\begin{array}{c}d_{1} \\ d_{2}\end{array}\right]$.

If faults occur during the work of the UM, it should be promptly detected and identified. Then the fault accommodation can be used. The traditional methods of diagnosis that focuses on systems described by linear models cannot provide the solution to the problem of synthesis of accommodation systems for electric servo actuators of the UM described by model with the presence of the nonlinear component $B(x)$.

In this paper, another synthesis method of fault accommodation systems is proposed. This method includes two basic steps: 1) using LDA to construct the diagnostic observers [12] with residual feedback [18] which can detect occurrence of specific faults and identification of their values; 2) forming of control signal which can provide fault tolerance to actuators of UM. Below these stages are considered in more detail.

\section{Synthesis of fault accommodation system}

The LDA includes following steps [12]. 1. Replacing the initial nonlinear system (3) by linear system. 2. Solving the accommodation problem for the linear system with some additional restrictions and obtaining the bank of linear observers. 3. Transforming the linear observers into the nonlinear ones. The feedback signals by residual are introduced in observers 
for fault identification [18]. The nonlinear diagnostic observers with residual feedbacks detecting the faults have following general form

$$
\begin{aligned}
& \dot{x}_{*}(t)=F_{*} x_{*}(t)+G_{*} u(t)+B_{*}\left(x_{*}\right)+V y(t)+w(r(t)), \\
& y_{*}(t)=N_{*} x_{*}(t),
\end{aligned}
$$

where $x_{*}(t) \in R^{k}$ is the state vector of the observer, $y_{*}(t)$ is the output signal, $F_{*} \in R^{k \times k}, G_{*} \in R^{k}, B_{*} \in R^{k}, V \in R^{k \times 2}$ and $N_{*} \in R^{k}$ are constant matrices, $k$ is dimension of the observer; $w$ is feedback vector.

It is assumed that the matrices $F_{*}$ and $N_{*}$ can be represented in a canonical form [12]: $F_{*}=\left[\begin{array}{ccccc}0 & 1 & 0 & \ldots & 0 \\ 0 & 0 & 1 & \ldots & 0 \\ . & . & . & . \\ 0 & 0 & 0 & \ldots & 0\end{array}\right]$, $N_{*}=\left[\begin{array}{lllll}1 & 0 & 0 & \ldots & 0\end{array}\right]$.

The observer (4) generates the residual $r(t)=C y(t)-y_{*}(t)$ for certain row matrix $C \in R^{2}$ which should be determined. If there are no faults and $d=0$, then $r(t)=0$, if a fault occurs, $r(t) \neq 0$. It is well-known [12] that for the observer design, the matrix $\Phi$ such that $\Phi x(t)=x_{*}(t)$ in the unfaulty case after the response to unlike conditions have died out plays the main role. In the absence of faults, the following well-known set of equations holds [12]:

$$
\begin{aligned}
& N_{*} \Phi=C N, \\
& \Phi F=F_{*} \Phi+V N, \\
& G_{*}=\Phi G, \\
& B_{*}\left(x_{*}\right)=\Phi B(x) .
\end{aligned}
$$

For each fault $d$, the diagnostic observer should be constructed. Matrices and vectors $\Phi, F_{*}, B_{*}\left(x_{*}\right), G_{*}, V$ and $C$ describing the observer can be found from (5). As a result, the observers $\mathrm{O}_{1}$ and $\mathrm{O}_{2}$ for the faults $d_{1}(t)$ and $d_{2}(t)$ were obtained:

$$
\begin{aligned}
& \mathrm{O}_{1}: \Phi=\left[\begin{array}{ccc}
1 & 0 & 0 \\
-1 & 0 & 0
\end{array}\right], G_{*}=\left[\begin{array}{l}
0 \\
0
\end{array}\right], V=\left[\begin{array}{cc}
1 & 1 / i_{r} \\
0 & -1 / i_{r}
\end{array}\right], C=\left[\begin{array}{ll}
1 & 0
\end{array}\right], B *=\left[\begin{array}{l}
0 \\
0
\end{array}\right] ; \\
& \mathrm{O}_{2}: \Phi=\left[\begin{array}{ccc}
0 & 1 & 0 \\
0 & R / L & k_{m} / J
\end{array}\right], G_{*}=\left[\begin{array}{c}
0 \\
\frac{k_{u} k_{m}}{L J}
\end{array}\right], V=\left[\begin{array}{cc}
0 & \left(-k_{v} / J\right)-(R / L) \\
0 & -k_{\omega} k_{m} / L J-k_{v} R / L J
\end{array}\right], C=\left[\begin{array}{ll}
0 & 1
\end{array}\right], \\
& B_{*}=\left[\begin{array}{c}
-\hat{M}^{*} / J \\
-\hat{M}^{*} R / J L
\end{array}\right] .
\end{aligned}
$$

For solving of identification problem for observers (6) which have dimension $k=2$ it is proposed to used approach developed in paper [18]. This approach uses residual signal feedbacks for elimination of mismatch vector between diagnosed object and observer, i.e. $e(t)=\Phi x(t)-x_{*}(t)=0$, even in the presence of faults. The procedure for the synthesis of such feedbacks is shown in detail in the paper [18].

After applying this known procedure [18] for observers $\mathrm{O}_{1}$ and $\mathrm{O}_{2}$ it is possible to receive the following feedbacks for fault identification:

$$
\begin{aligned}
& w=\left[\begin{array}{ll}
w_{1} & w_{2}
\end{array}\right]^{T}, \\
& w_{1}=\frac{T_{1}}{T_{0}} r(t), \\
& w_{2}=\frac{T_{2}}{T_{0}} r(t)+\int \frac{1}{T_{0}} r(t) d t,
\end{aligned}
$$

where $T_{0}, T_{1}$ and $T_{2}$ are feedback coefficients. These coefficients are selected in such a way as to guarantee stability to observers. Finally, it is possible to identify the faults value for the electric servo actuators of the UM [18]: 


$$
\begin{aligned}
& \tilde{a}_{r}=-\frac{1}{f_{1} T_{0}} \iint r d t, \\
& \tilde{k}_{v}=-\frac{J}{f_{2} T_{0} \dot{\alpha}} \int r d t,
\end{aligned}
$$

where $f_{1}=-1$ and $f_{2}=R / L$. Thus, the problem of identification of value of faults has been solved.

The next step of synthesis of the fault accommodation system is forming a control law for the compensation of the detected fault. Actuators of the UM are described by nonlinear differential equations with variable parameters. The control law for such objects can be formed using an approach of synthesis of corrective devices, which provides stabilization of the parameters of the control object to the nominal values [19].

At first the desired dynamic properties of actuators of the UM should be defined. Dynamic properties for actuators with DC motors can be defined by the equation $L J \dddot{\alpha}+R J \ddot{\alpha}+k_{\omega} k_{m} \dot{\alpha}=k_{u} k_{m} \tilde{u}$, which is the differential equation with constant coefficients, where $\tilde{u}$ is a control signal of adaptive regulator.

Next, variable $\dddot{\alpha}$, which is the highest derivative of the object output signal, should be expressed in this equation. Desired control law can be obtained by the substitution of this variable into the initial model of actuator. Thus, control law obtained by this method is a function of the output of the system and its lower derivatives. Control law of this form ensures the required performance and dynamic properties of the actuators of the UM. For stabilizing the properties of actuators in case of faults, estimates of these faults obtained by observers (6) with feedbacks (7) should be used in the control law. Thus, the accommodation to faults in actuators of the UM can be achieved by the following control law:

$$
u=P(\varepsilon)\left[a_{r}^{d}-a_{r}+\tilde{a}_{r}\right]+\frac{L\left(k_{v}+\tilde{k}_{v}\right)}{k_{u} k_{m}} \ddot{\alpha}+\frac{R\left(k_{v}+\tilde{k}_{v}\right)}{k_{u} k_{m}} \dot{\alpha}+\frac{R}{k_{u} k_{m}}\left[\hat{M}^{*}+M_{c f}\right]+\frac{L \dot{\hat{M}}^{*}}{k_{u} k_{m}},
$$

where $P(\varepsilon)$ is a function of typical regulator, and $a_{r}^{d}$ is a desired position of the output shaft of actuator reduction gear.

Block diagram of the actuator with the synthesized fault accommodation system is shown in Fig. 2.

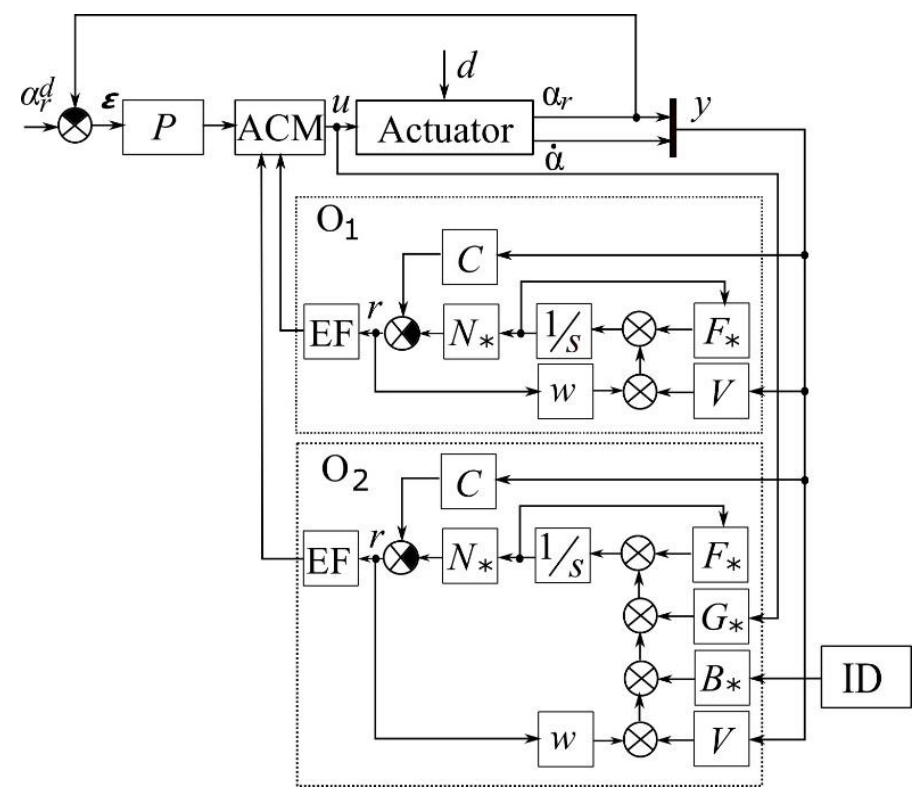

ACM - accommodation block (8), EF - block of estimation of faults value (7), ID - block of solving inverse dynamics problem of UM (1)

Fig. 2. Block diagram of the actuator with the synthesized fault accommodation system

The synthesis of proposed control law (8), which provides accommodation to faults for all actuators of the UM, is quite simple. Such control law can be realized on the base of common microcontrollers. In such way, it is possible to provide accurate stabilization of the dynamic properties of the actuators of underwater multilink manipulators described by complicated differential equations with variable parameters and nonlinearity, by using additional signals of residuals in the law of self-tuning, even in the presence of a fault.

4. Simulation of performance of fault accommodation system 
Analysis of effectiveness of developed fault accommodation system was implemented using model of UM with three degrees of freedom presented on Fig. 3. The accommodation system was synthesized for electric servo actuator of second degree of freedom of this UM. The results for other degree of freedom of the UM are similar.

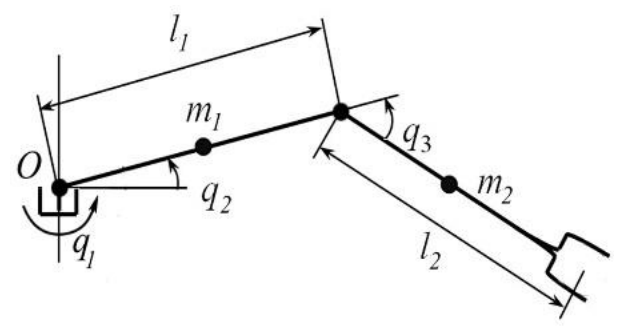

Fig. 3. The kinematic scheme of UM with 3 degrees of freedom

Parameters of UM have the following values: $l_{1}=l_{2}=0.5 \mathrm{~m}$ are lengths of UM links; $m_{1}=m_{2}=3.9 \mathrm{~kg}$ are masses of links. The links centers of size coincide with their centers of weights. Links have neutral buoyancy and cylindrical form with base radius $r_{1}=r_{2}=0.05 \mathrm{~m}$. Tensors of inertia of these links concerning their centers of weights are diagonal and $\tau_{1_{11}}=\tau_{2_{11}}=5 \cdot 10^{-3}, \tau_{1_{22}}=\tau_{1_{33}}=\tau_{2_{22}}=\tau_{2_{33}}=8.4 \cdot 10^{-2}$ are elements of inertia tensors of the first $\tau_{1}$ and second $\tau_{2}$ links, respectively. Besides $\Pi_{1}=\Pi_{2}=1.075 \mathrm{~kg} ; \quad\left|r_{1}^{*}\right|=\left|r_{2}^{*}\right|=0.25 \mathrm{~m} ; \quad K_{A 2}=\sin \psi_{2} ; \rho=10^{3} \mathrm{~kg} / \mathrm{m}^{3}$; $k_{L 1}=k_{L 2}=0.012 ; \quad k_{1}^{*}=k_{2}^{*}=0.89 ; T_{1_{11}}=T_{2_{11}}=1.4 \cdot 10^{-3} ; T_{1_{22}}=T_{1_{33}}=T_{2_{22}}=T_{2_{33}}=2.6 \cdot 10^{-2}$ are diagonal elements of inertia tensors of fluid masses attached to appropriate UM links.

The electric drive servo actuator is described by following set of parameters: $R=0.5 \Omega, L=0.0005 \mathrm{H}, k_{\omega}=0.04 \mathrm{~V} \cdot \mathrm{s}$, $k_{m}=0.04 \mathrm{~N} \cdot \mathrm{m} / \mathrm{A}, J=10-3 \mathrm{~kg} \cdot \mathrm{m}^{2}, i_{r}=100, k_{v}=0.005 \mathrm{~N} \cdot \mathrm{m} \cdot \mathrm{s} / \mathrm{rad}, M_{c f 0}=0.02 \mathrm{~N} \cdot \mathrm{m}, k_{u}=800$.

The input signal $q_{2}=\alpha_{r}^{d}=\sin (1.5 t)$ was applied to the electric servo actuator. The parameters of feedbacks for observers were chosen as follows: $T_{0}=4.63 \cdot 10^{-6}, T_{1}=8.33 \cdot 10^{-4}, T_{2}=0.05$.

Faults were presented by changing the following values:

- adding the error of position sensor signal $\tilde{a}_{r}=0.1 \mathrm{rad}$ at the time $t_{1}=3 \mathrm{~s}$;

- increasing viscous friction coefficient by $50 \% \tilde{k}_{v}=0.0025 \mathrm{~N} \cdot \mathrm{m} \cdot \mathrm{s} / \mathrm{rad}$ at the time $t_{2}=5 \mathrm{~s}$.

Values of introduced faults (curve 1) and estimated value of fault (curve 2) are shown on the Fig. 4 and Fig.5. It follows from these figures that the constructed system provides accurate estimation of occurred faults. Fig. 6 shows the value of dynamic error of position of actuator in absence (curve 1) and presence (curve 2) of faults. Fig. 7 shows the value of dynamic error of position of actuator with accommodation system (curve 1) and without accommodation system (curve 2).

The results of simulation confirm the efficiency of the proposed fault accommodation system for actuators of UMs. Realization of the synthesized accommodation systems does not present any difficulties. These systems can be realized with the help of serial microprocessors.

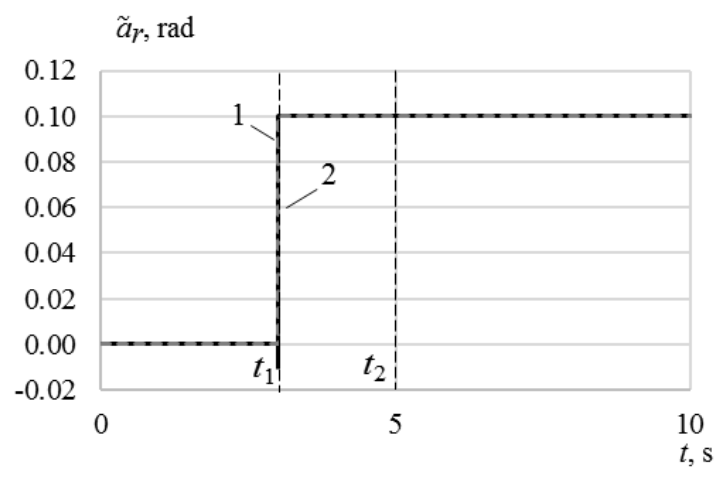

Fig. 4. Estimation of fault $\tilde{a}_{r}$ value by proposed fault accommodation system

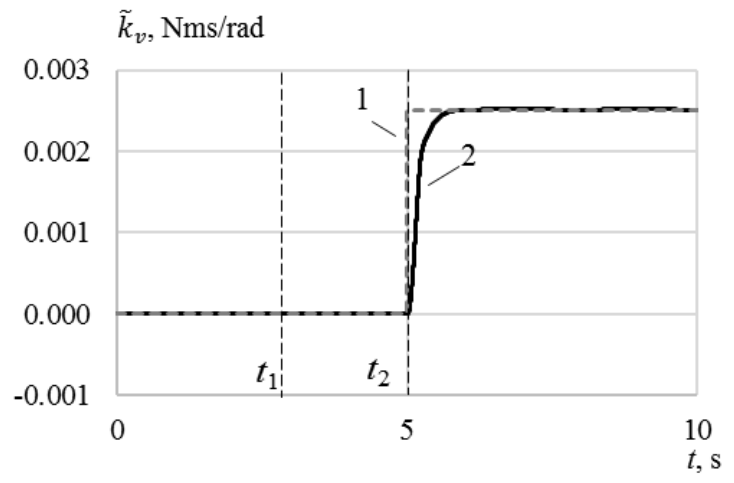

Fig. 5. Estimation of fault $\tilde{k}_{v}$ value by proposed fault accommodation system 
$\varepsilon, \operatorname{rad}$

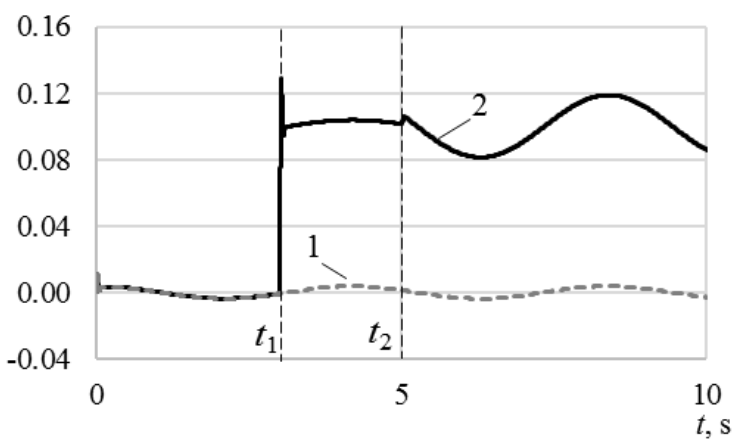

Fig. 6. Dynamic error of position of actuator in absence and presence of faults $\varepsilon, \mathrm{rad}$

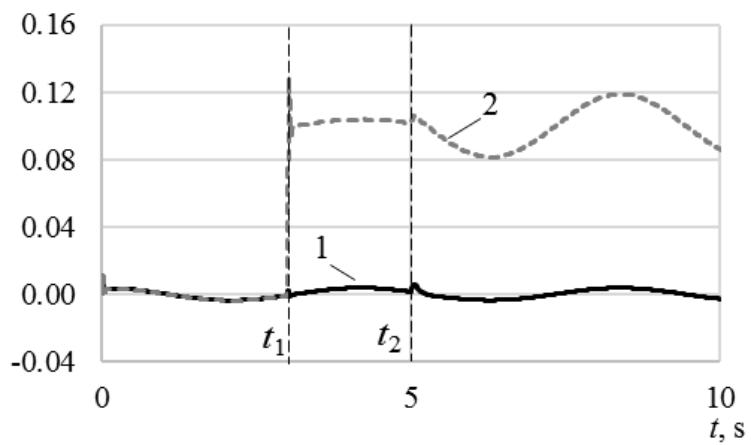

Fig. 7. Dynamic error of position of actuator with fault accommodation system and without fault accommodation system

\section{Conclusion}

An effective fault accommodation system for electric servo actuators of UMs is suggested. Two typical faults in actuators of UM were considered: fault caused by changing of viscous friction moment and error in the sensor, which measures the position of output shaft of gear. For diagnosis and identification of these faults were synthesized observers with residual signal feedbacks. It was proposed to realize the accommodation to faults by generation of additional control signals based on identified value of faults. As a result, it is possible provide the reliability of underwater working of manipulators in the event of faults.

The mathematical modeling confirms the efficiency of proposed fault accommodation system for actuators of UMs. The future work will relate to solving of problem of exact fault identification at the acting of unknown disturbance.

\section{Acknowledgment}

This research was supported by grant of President of Russia (MK-8536.2016.8) and grant of RFBR (16-29-04195), 17-57-45055).

\section{References}

[1] Wynn, R., Huvenne, V., Le Bas, T., Murton, B. and et al. (2014). Autonomous Underwater Vehicles (AUVs): Their past, present and future contributions to the advancement of marine geoscience. Marine Geology, Vol.352, (2014), pp. 451-468, ISSN 0025-3227.

[2] Bogue R. (2015). Underwater robots: a review of technologies and applications. Industrial Robot: An International Journal, Vol. 42, No. 3, (2015), pp.186-191, ISSN 0143-991X.

[3] Kim, T.W., Marani, G. \& Yuh, J. (2016). Underwater vehicle manipulators, In: Handbook of Ocean Engineering, Dhanak M.R., Xiros N.I. (Ed.), pp. 407-422, Springer, ISBN 978-3-319-16648-3.

[4] Yuh, J., Marani, G. \& Blidberg, D. (2011). Applications of marine robotic vehicles. Intelligent Service Robotics, Vol. 4, No. 4, (2011), pp. 221-231, ISSN 1861-2776.

[5] Filaretov, V. \& Konoplin, A. (2016). Development of control systems for implementation of manipulative operations in hovering mode of underwater vehicle, Proceedings of OCEANS 2016, 2016, Shanghai, China, ISBN 978146739724-7, pp. 1-5, DOI: 10.1109/OCEANSAP.2016.7485692.

[6] Filaretov, V.; Konoplin, A; Konoplin, N. \& Gorbachev, G. (2016). Control system for underwater vehicle with multilink manipulator for automatic manipulation operations, Proceedings of the 27th DAAAM International Symposium, pp.0714-0720, B. Katalinic (Ed.), Published by DAAAM International, ISBN 978-3-902734-08-2, ISSN 1726-9679, Vienna, Austria, DOI: 10.2507/27th.daaam.proceedings.103.

[7] Blanke, M.; Kinnaert, M.; Lunze, J. \& Staroswiecki, M. (2003). Diagnosis and Fault Tolerant Control, SpringerVerlag, ISBN 978-3-540-35652-3, Berlin, Heidelberg.

[8] Frank, P. (1990). Fault diagnosis in dynamic systems using analytical and knowledge-based re-dundancy - A survey and some new results, Automatica, vol. 26, pp. 459-474.

[9] Schreier, G.; Ragot, J.; Patton, R. \& Frank, P. (1997). Observer design for class of nonlinear systems, Proceedings of the IFAC Symposium SAFEPROCESS'97, UK, Hull, pp. 498-503.

[10] De Persis, C. \& Isidori, A. (2001). A geometric approach to nonlinear fault detection and isolation, IEEE Trans. Automat. Contr., Vol. AC-46, No. 6, pp. 853-865. 
[11] Shumsky, A.Ye. \& Zhirabok, A.N. (2006). Nonlinear diagnostic filter design: Algebraic and geo-metric points of view, Int. J. Applied Mathematics and Computer Science, Vol. 16, No. 1, pp. 115-127.

[12] Zhirabok, A. \& Usoltsev, S. (2000). Linear diagnosis methods for nonlinear systems, Automation and Remote Control, Vol. 7, pp. 149-159.

[13] Isermann, R. (2006). Fault-Diagnosis Systems: An Introduction from Fault Detection to Fault Tolerance, SpringerVerlag, Berlin.

[14] Staroswiecki, M.; Yang, H. \& Jiang, B. (2006). Progressive accommodation of aircraft actuator faults, Proceedings of IFAC Symposium Safeprocess, Beijing, China, pp. 877-882.

[15] Filaretov, V.F.; Zhirabok, A.N.; Zuev, A.V. \& Procenko, A.A. (2013). Synthesis Method of Fault Tolerant Control System for Manipulators, Advanced Materials Research, Vol. 717, pp 551-556.

[16] Filaretov, V.F.; Zirabok, A.N.; Zuev, A.V. \& Protsenko, A.A. (2012). The Development of the Faults Accommodation System for Actuators of Multilink Manipulator, Proceedings of the 23th DAAAM International Symposium, pp.575-578, B. Katalinic (Ed.), Published by DAAAM International, ISSN 1726-9687, ISBN 978-3901509-86-5, Zadar, Croatia.

[17] Filaretov, V.F. \& Konoplin, A.Yu. (2015). System of Automatic Stabilization of Underwater Vehicle in Hang Mode with Working Multilink Manipulator, International Conference on Computer, Control, Informatics and Its Applications, Bandung, Indonesia, pp. 132-137.

[18] Filaretov, V.; Zhirabok, A.; Zuev, A.; Protcenko, A. (2016). Fault identification in nonlinear dynamic systems, Proceedings of the 5th International Conference on Systems and Control, Marrakesh, Morocco, pp. 273-277.

[19] Filaretov, V.F. (2007). Synthesis of Adaptive Control Systems for Electric Servo Actuators of Manipulators, Proceedings of the 28th DAAAM International Symposium, pp. 277- 279, B. Katalinic (Ed.), Published by DAAAM International, ISSN 1726-9679, ISBN 3-901509-58-5, Zadar, Croatia. 